\title{
Increased Functional Connectivity Characterises the Prodromal Phase of the Migraine Cycle
}

\author{
Anne Stankewitz ${ }^{1,2}$, Astrid Mayr ${ }^{3}$, Enrico Schulz ${ }^{1,4}$ \\ ${ }^{1}$ Department of Neurology, University Hospital LMU, Ludwig-Maximilians-Universität \\ München, Munich, Germany \\ ${ }^{2}$ Department of Neurology, Klinikum rechts der Isar, Technische Universität München, \\ Munich, Germany. \\ ${ }^{3}$ Department of Radiology, University Hospital LMU, Ludwig-Maximilians-Universität \\ München, Munich, Germany \\ ${ }^{4}$ Department of Medical Psychology, Ludwig-Maximilians-Universität München, Munich, \\ Germany
}

Running title: Connectivity across migraine cycle

Keywords: Episodic migraine; functional connectivity; fMRI; prodromal phase; migraine cycle

Abbreviations: $\mathrm{EP}=$ evoked potentials; $\mathrm{CNV}=$ contingent negative variations; $\mathrm{FC}=$ functional connectivity; ROI = region of interest; $L M E=$ linear mixed effects model; ICA = independent component analysis; $\mathrm{PAG}=$ periaqueductal grey; $\mathrm{FD}=$ framewise displacement; $\mathrm{PCA}=$ principal component analysis; $\mathrm{PCC}=$ posterior cingulate cortex; $\mathrm{ACC}=$ anterior cingulate cortex; AAIC = anterior agranular insular cortex; LC = locus coeruleus; BA = Brodmann area

Corresponding authors:

Anne Stankewitz, Enrico Schulz

Department of Neurology

Ludwig-Maximilians-Universität München

Fraunhofer Str. 20

82152 Martinsried, Germany

Email: as@pain.sc,es@pain.sc 


\section{Abstract}

Episodic migraine is reflected by cyclic changes in behaviour and cortical processing. In this study, we aimed to identify how functional connectivities change over the entire migraine cycle.

By using longitudinal neuroimaging and a whole-brain analysis approach, we tested 12 episodic migraine patients across 82 fMRI recordings during spontaneous headache attacks, with follow-up measurements over the pain-free interval without any external stimulation.

We focussed on two different trajectories with a linear increase of connectivity and a peak either during the prodromal phase or during the headache attack. We found cycle-related connectivity changes for the anterior insula and the posterior orbitofrontal cortex. Both regions showed the highest connections with sensory, motor, and cingulate areas in the prodromal phase. The connectivity between the hypothalamus and the locus coeruleus was strongest during the headache attack.

The peak connectivity during the prodromal phase and its collapse during the headache can be regarded as a mechanism of normalising cortical processing. The increase of synchronicity of functional connections during the ictal phase of the migraine cycle may contribute to the variety of symptoms of migraine attacks, such as headache, hypersensitivity to sensory modalities, and autonomous symptoms. We speculate about a malfunction at the molecular level in agranular frontal and insular brain regions, which needs to be followed up upon in subsequent studies.

\section{Abbreviated Summary}

Episodic migraine is a cyclic disease. We investigated functional connectivities over an entire migraine cycle. We found cycle-related connectivity changes for two proximate agranular regions. The prodromal increase and the collapse of connectivity during the headache may reflect normalising cortical processing. 


\section{Introduction}

Episodic migraine is a cyclic disease that affects about $12 \%$ of the global population ${ }^{1}$. Although recurring headache episodes are often the most disabling symptom, migraine attacks are more than just a headache; attacks typically last up to 72 hours and contain up to four phases with characteristic symptoms separated by interictal intervals with a variable length: the prodromal (premonitory), aura, headache, and postdrome phase ${ }^{2,3}$.

The cyclic nature of migraine disease. Evidence for cyclic changes in the migraineurs' brain originates from early neurophysiological studies: EEG results revealed a lack of habituation to repetitive sensory stimulation ${ }^{4}$ and altered amplitudes of contingent negative variation (CNV) abnormalities ${ }^{5}$ during the pain-free interval that normalised just before or during the headache attack ${ }^{6}$. Migraine phase-dependent BOLD activity in the trigeminal nuclei ${ }^{7}$ and the hypothalamus ${ }^{8-10}$ has further been observed in FMRI studies in response to trigeminal painful stimulation ${ }^{11}$. In addition, enhanced functional connectivities (FC) were observed between limbic regions during the preictal phase and between the pons and the hypothalamus during the headache attack $\mathrm{e}^{11}$. During the headache attack, FC alterations have been reported in pontine regions ${ }^{12}$ and the thalamus ${ }^{13}$. Recently, our group observed cyclic changes of sensory, thalamic and sensory networks: the activity within these networks linearly increased over the pain-free interval, with peak activity during the prodromal phase, but a decoupling of network regions during the acute headache attack 14.

Hypothalamus as a rhythmic generator of migraine attacks. The cyclicity of episodic attacks in migraineurs has been attributed to the hypothalamus due to its role in the regulation of biological rhythms and in maintaining internal homeostasis by controlling the endocrine and the autonomic nervous system ${ }^{15,16}$. Several migraine symptoms and trigger factors of attacks are related to the hypothalamus. Hypothalamic alterations were indeed observed not only during the headache attack ${ }^{17}$, but also prior to the headache and during the prodromal phase ${ }^{8-10}$.

The importance of the preictal migraine phase. Previous research ${ }^{4,8-10}$ as well as clinical features ${ }^{15}$ of the disease (e.g. attack trigger and premonitory symptoms) suggest that the initiation of headache attacks most likely starts during the preictal - pain-free - interval, which may be hours or even days before the headache occurs. However, most imaging studies on migraine were utilising cross-sectional designs; longitudinal within-subject designs, which are necessary to shed light on the cyclicity of migraine-related mechanisms in the brain, are rare ${ }^{9,18}$. In addition, connectivity studies which investigated patients at different migraine phases used a priori-defined seed regions instead of taking the whole-brain connectivity into account ${ }^{19}$.

To expand our knowledge about the cyclic mechanisms of the migraineurs' brain, we aimed to explore the trajectory of whole-brain FC during resting state-fMRI (rs-FMRI) over the entire migraine cycle. First, based on the clinical picture of the disease and previous imaging findings, we hypothesised hypothalamic alterations in connectivity along the 
migraine cycle. Second, due to sensory dysfunctions reported by migraine patients during and outside attacks, we hypothesised cyclic dysfunctions in functional connections involving sensory brain areas.

\section{Materials and Methods}

\section{Subjects}

12 patients with episodic migraine (11 females and one male) were included in the study (Supplementary Table 1). The time points of the attacks were equally distributed in reference to the menstrual cycles of the female patients. Migraine diagnosis was based upon the classification of the International Headache Society ${ }^{20}$. The patients did not report any other neurological or psychiatric disorders, were not taking preventative medication for migraine for at least six months but were allowed to take their regular acute migraine medication after the recording of the headache attack (non-steroidal anti-inflammatory drugs or triptans). Due to their effects on cortical perfusion, patients were not permitted to consume coffee or other caffeinated beverages. All patients gave their written, informed consent. The study was conducted according to the Declaration of Helsinki and approved by the Ethics Committee of the Technische Universität München, Germany. All patients were remunerated for participation.

\section{Image Acquisition}

MRI data were collected on a 3 tesla scanner (Ingenia, Philips, The Netherlands) using a 32-channel head coil. Patients were instructed to remain awake and relaxed with their eyes closed. For the 300 volumes of resting state data, we used the following parameters: TR = $2000 \mathrm{~ms} ; \mathrm{TE}=30 \mathrm{~ms} ; \mathrm{FOV}=192 \times 192 \mathrm{~mm}^{2}$; flip angle $=90^{\circ}$; number of slices $=37$; voxel size $=3 \times 3 \times 3 \mathrm{~mm}^{3}$ (0.29 mm gap). For image registration, a high resolution T1-weighted anatomical image was collected with: $\mathrm{TR}=9000 \mathrm{~ms}, \mathrm{TE}=4 \mathrm{~ms}$, flip angle $=8^{\circ}, \mathrm{FOV}=240 \mathrm{x}$ $240 \times 170 \mathrm{~mm}^{3}$; number of slices $=170$; voxel size $=1.0 \times 1.0 \times 1.0 \mathrm{~mm}^{3}$ ). Field maps were acquired in each session to control for B0-effects; 64 slices, TR $=960 \mathrm{~ms}$, FOV $=192 \times 192$ $\mathrm{mm}^{2}$; voxel size $=2.0 \times 2.0 \times 2.0 \mathrm{~mm}^{3}, 0.2 \mathrm{~mm}$ gap between slices. TE $=6 \mathrm{~ms} / 10.55 \mathrm{~ms}$, flip angle $60^{\circ}$.

\section{Study Design}

Migraine patients were tested repeatedly over an entire migraine cycle (Figure 1). The imaging time series for each patient started with the recording of a spontaneous and untreated headache attack within the first 6 hours after the beginning of the headache. We only recorded headache attacks which were reported with an intensity of moderate to severe, predefined as a minimum of " 4 " on a numerical rating scale with the endpoints " 0 " (no pain) and "10" (extremely intense pain). Brain data were then recorded every 1-4 days at the same time of the day until patients informed us by phone about the following headache attack (which was not scanned) and the time series was completed with the last attack-free recording. 


\section{Image Preprocessing}

The data were preprocessed with FSL. The Melodic toolbox was used to execute brain extraction, high-pass filtering with a frequency cutoff of $1 / 100 \mathrm{~Hz}$, spatial registration to the MNI template, corrections for head motion during scanning, and spatial smoothing (5mm FWHM). A distortion correction of the images was used based on field maps. The data were further semi-automatically cleaned of artefacts with an independent component analysis (ICA) through Melodic ${ }^{21}$. The number of components had been automatically estimated by the software and artefact-related components were removed from the data according to the recommendations of Griffanti and colleagues ${ }^{22}$. Head movement during scanning did not exceed $2 \mathrm{~mm}$ or $2^{\circ}$ in any direction.

The time series of functional volumes were projected to surface space by using the "Connectome Workbench" package ${ }^{23}$. Regions of interest (ROIs) were defined by subdividing the cortical surface into 180 regions per hemisphere ${ }^{24} .65$ further regions including the cerebellum and subcortical regions, such as the periaqueductal grey (PAG), the thalamus, cerebellar subregions, and the amygdala, were also included. The time courses for all voxels of cortical activity for a specific region of the Glasser Atlas, e.g. the middle insula, were extracted. Nuisance variables (McFLIRT motion parameters, their squares and temporal derivatives with squares; total of 24 regressors), as well as outliers, were regressed out from the data. Outliers in the FMRI data are defined by the framewise displacement (FD) and DVARS following Power and colleagues ${ }^{25}$. A volume is defined as an outlier if it exceeds one of the following thresholds: FD $\geqq 0.2 \mathrm{~mm}$ or DVARS $\geqq$ (the 75 th percentile +1.5 times the InterQuartile Range). Outliers are marked for each subject and then included in the regression as a vector of zeros (non-outlier) and ones (outlier). We computed principal component analyses (PCA) separately for each ROI and subject and selected the first component (Version R2018a, Mathworks, USA). Time courses of the 425 components were correlated using Kendall's $\tau$ coefficient.

\section{Statistical Analyses}

We explored the cyclic change of cortical resting-state connectivity between the 425 regions over the migraine interval. To investigate how the cortical connectivities evolve over the trajectory of measurements, we computed linear mixed effects models (LME) for each pair of brain regions and related the time points within the migraine cycle to the strength of connectivity quantified by Kendall's $\tau$. We created a time vector for each patient's migraine cycle and encoded the day of the recording by assigning numbers between 1 and 2 . The numbers 1 or 2 were assigned to the measurement during the headache attack, depending on the following two different trajectories of map strengths in the brain ${ }^{9}$ :

Trajectory 1 ("reset mode"): In this model, we aimed to detect the resting-state connectivity that has its peak (positive or negative) just before the headache attack starts and then drops to the level (positive or negative) during the headache. From here, the map strength linearly increases (or decreases) over the migraine cycle to the next attack. In this hypothetical time course, the map strength on the first day after the attack would be 
similar to the brain connectivity during the attack. This trajectory can be interpreted as a cortical "reset" mechanism and is in line with neurophysiological studies reporting a habituation deficit in pain-free migraineurs that normalise during headache attacks ${ }^{26}$. For 5 measurements over 10 days, the following vector is used to reflect the trajectory 1: 1 (=attack), 1.2, 1.4, 1.6, 1.8.

Trajectory 2 ("pain mode"): In this model, we aimed to detect the resting-state connectivity that has a peak during the headache attack and drops to the level (positive or negative) the following day. From there, we assume a linear increase (or decrease) over the migraine cycle towards the next attack. We hypothesise increased brain connectivity in regions that contribute to the processing of migraine symptoms, e.g. pain, increased sensitivity to light, sound, and odours, and vegetative complaints. In this hypothetical time course, the brain connectivity on the day prior to the attack would be similar to the brain connectivity during the headache attack and is suggested to reflect the steadily increasing excitability of the brain. Similar to the above-mentioned example with 5 measurements over 10 days, the following vector is used for trajectory 2: 2 (=attack), 1.2, 1.4, 1.6, 1.8.

The statistical analysis for the trajectory of resting-state connectivity has been performed in Matlab. To explore the relationship between the fluctuating map strength and the variable pain experience, we computed $\mathrm{LMEs}^{27}$ that related the longitudinal recordings of resting-state connectivity to the number vector of recording days:

\section{f_connectivity cycle $+(1 /$ subject $)$}

The model is expressed in Wilkinson notation ${ }^{28}$; the included fixed effect (f_connectivity cycle) essentially describes the magnitudes of the population common intercept and the population common slope for the dependency of cortical data on the factor time. The added random effect (e.g. 1 | subject) is used to model the specific intercept differences for each subject. In other words, the model estimates the relationship between the cortical processes in dependence on their occurrence in the migraine cycle (fixed effect). Please note that the statistical terms of fixed effect and random effect are differently used in the common neuroimaging software package SPM as compared with the standard nomenclature in statistics that we are following here. T-values are computed voxel-wise as quotients between the beta estimates and the standard errors of the equation. For a comprehensive introduction to LMEs, see ${ }^{29}$. Based on 5000 randomisations, the statistical thresholds were determined using the "palm_datapval.m" function publicly available in PALM ${ }^{30,31}$. Connectivity figures were generated using NeuroMArVL (link). 


\section{Results}

To determine the cyclic changes of the functional connectivity in the migraineurs' brain between two migraine attacks, we computed linear mixed effects models (LME) and analysed how the resting-state functional connectivity is evolving depending upon the time point within the migraine cycle. The findings are presented in Figures 3a, 3b, and 3c. A confusion matrix gives a more global impression on the direction of connectivity changes throughout the migraine cycle (Figure 3a), a circular plot shows all significant connectivities (3b), and the best-connected brain regions are presented on a 3D brain (3c). All figures show different aspects of the same underlying results. Two possible time courses were explored:

Trajectory 1. For this trajectory, the functional connectivity between brain regions increased/decreased linearly over the interictal interval towards the attack, reached its peak/trough prior to the beginning of the headache, and dropped back to normal during the headache. We found three larger complexes of brain regions that account for most of the significant connections: the insular cortex and surrounding regions, the dorsal posterior cingulate cortex (PCC; Brodmann area 31), and the dorsal anterior cingulate cortex (ACC; Brodmann area 24 and 32).

Insula and adjacent interior frontal cortex. For the insular cortex and its surrounding sub-regions, the most affected region is the area $47 \mathrm{~m}$ (12 connections), which drops its connection during the migraine attack to insular and parietal opercular regions, the primary somatosensory cortex, regions that process eye movements, and the dorsal posterior cingulate cortex. The anterior agranular insular cortex (AAIC; 10 connections) exhibits a steady increase of connectivity prior to the attack and shows a drop of connectivity predominantly to areas related to sensory functions (auditory - lateral belt region, primary auditory cortex; visual - V3, V3A, V4t, V6A; visual integration areas - V6A, TPOJ, superior temporal visual area). Additionally, we found cyclic connectivity changes of the thalamus to the right posterior insular cortex.

Posterior Cingulate Cortex. We found several connections between the PCC (BA 31) and the insular-opercular regions.

Anterior Cingulate Cortex. The dorsal ACC showed multiple connections to the inferior frontal regions (BA 47), visual (V1 to V3), and higher-order visual areas (prostriate and ventromedial visual areas 1 and 2 ).

Trajectory 2. For trajectory two, we found a single significant effect which is the connectivity between the hypothalamus and the pons, anatomically localised at the locus coeruleus (LC). This connectivity linearly increased over the interictal interval towards the next attack, exhibited its highest strength during the headache phase, and dropped thereafter. 


\section{Discussion}

The present longitudinal FMRI study aimed to detect the whole-brain functional connectivity in episodic migraineurs over the migraine cycle. By taking the time point of the attack into account, we analysed two possible connectivity trajectories: (1) a linear trajectory (increase or decrease) towards the attack with peak connectivity just before the attack, followed by connectivity "reset" to the baseline level during the headache ("reset mode"), or (2) a linear trajectory (increase or decrease) towards the attack with peak connectivity during the headache ("pain mode"). Here, we found three major brain regions that followed the "reset mode": the insular cortex and its surrounding sub-regions, the dorsal PCC, and the dorsal ACC. In contrast, a single connection between the hypothalamus and the LC followed the trajectory of the "pain mode" by exhibiting the highest connectivity during the headache.

\section{Trajectory of the "reset mode"}

The fact that we found the majority of the dynamics of connectivity changes in the prodromal phase underlines the potential impact of the current findings on the clinical understanding of the disease. In line with the current view on the cyclic nature of migraine, the present study suggests that pathological processes are developing early on and can be observed and potentially treated before the painful phase of the headache attack is initiated. Our findings for this trajectory is in line with previous neurophysiological research suggesting a lack of habituation to sensory input during the interictal phase with a "normalisation" of cortical processes just before and during the headache attack ${ }^{4,5}$. Here, we identified three major regions that may represent these deviances.

Connectivity of insular regions. The connections with the right insular cortex are affected throughout the migraine cycle. On the one hand, we found the agranular anterior insular cortex (AAIC) to exhibit a steady increase of connectivity towards the attack and shows an ictal drop of connectivity particularly to sensory areas (auditory, visual). As the AAIC is also anatomically connected to olfactory areas, the region has been related to the processing and integrating of sensory information ${ }^{32}$. The anterior insula is also involved in the cognitive aspects of processing pain ${ }^{33,34}$ and in maintaining working memory functions in mice ${ }^{35}$ and men ${ }^{36}$.

This is in line with our previous work showing a similarly increasing connectivity from the hypothalamus to the right anterior insular cortex ${ }^{9}$. We suggested that the steadily increasing hypothalamic-insular connectivity would serve as a control mechanism for the activity in insular sub-areas. However, this hypothalamic control may require a steadily increasing effort over the migraine cycle, probably due to the increasing connectivity from sensory areas to the anterior insula that we find in the present investigation.

Connectivity of inferior frontal region $47 \mathrm{~m}$. The best-connected region is the right area $47 \mathrm{~m}$, which is located in the inferior frontal cortex. Due to its proximity to the insular cortex, the pain-related effects in this region can be easily overlooked (e.g. Figure $2 \mathrm{C}$ in ${ }^{37}$ ). An involvement of this region in the context of pain and migraine has been found in the seminal work of Denuelle and colleagues ${ }^{17}$. Using PET during migraine attacks, the 
Brodmann Area 47 was active during the headache as well as after the successful treatment of the headache with analgesic medication, suggesting that this region is involved in the underlying migraine-related cortical processes. Interestingly, due to concomitant hypothalamic findings, this region has not caused much attention in this publication.

In the present study, the area $47 \mathrm{~m}$ exhibits a migraine-related cyclic trajectory predominantly with regions in the left insula and with the bilateral posterior section of the anterior cingulate cortex (BA 32). There is a vast amount of literature showing the involvement of this cingulate region in the processing of pain ${ }^{38,39}$. However, the patients did not experience any headache pain at the peak connectivity of this region. The area $47 \mathrm{~m}$ also showed some cyclic connections to subregions in the BA6, which are mostly associated with premotor functions. While these motor functions remain to be interpreted, one of the subregions of BA6 (area PEF) is connected to the entorhinal cortex, which is processing olfactory information. Here, we may see a functional distinction in the migraine-related impairments of sensory processing (i.e. the lack of habituation). While visual and auditory processes are directly bound to the anterior insula cortex, migraine-related olfactory issues could be related to loops that are mediated by BA 47. In a similar vein, the area $47 \mathrm{~m}$ also exhibits connections to the posterior part of the ACC, which in turn connects with higher-order visual areas. This may suggest that the influence of the area $47 \mathrm{~m}$ on sensory processing is rather indirect and requires the mediation of other cortical regions (see link).

Cingulate connectivities. In addition, there are further cycle-related changes in connectivity between the posterior part of the ACC and the higher-order ventromedial visual areas (VMV). This subregion of the ACC has been associated with salient sensory stimulation ${ }^{40,41}$. The present findings suggest an impairment of sensory processing in the brain at several stages of the processing stream; the impairment includes lower-order and higher-order visual regions. The number of distinct connections between insular regions and posterior cingulate regions is surprising as both regions are known to be involved in the processing of the perception of pain ${ }^{39,42}$. However, the peak connection occurred just before the headache. The finding may suggest that the increase of connectivity may represent precursor activities for the headache in the following day(s). The finding may also represent the overly salient processing of sensory information ${ }^{40,41}$ throughout the migraine cycle due to the lack of habituation ${ }^{43}$. This is also in line with the working memory function of area $47 \mathrm{~m}^{44}$, which is connected to the posterior part of the ACC.

Normalisation of functional connectivity during the migraine attack. Taken together, the strong involvement of the regions aAIC and $47 \mathrm{~m}$ in sensory and working memory processes suggest the current view on migraine as an overflow of sensory input that can not be sufficiently emptied from cortical loops that maintain conscious processes. This is in line with neurophysiological findings that interictal migraine patients suffer from the inability to habituate to sensory stimulation in the interictal phase ${ }^{4,43,45}$. Similar to the connectivity of the aAIC and the BA47, habituation in migraineurs is reset to normal during and through a migraine attack ${ }^{4,46}$. Interestingly, the AAIC and the posterior part of the 
orbitofrontal cortex are similar regarding gross morphology and architecture ${ }^{47}$; they lack a granular layer 4 and are characterised by a substantial amount of von Economo neurons (VENs ${ }^{32,48}$ ). In their original work, von Economo and Koskinas (1925) stated that VENs can be found in "cap, corners and interior wall of the frontal part of the Gyrus limbicus, in the posterior transitional gyrus of the orbital part of the frontal lobe (which runs to the anterior frontoorbital Insula), in the so-called Gyrus transversus insulae and its associated Gyri breves accessorii (anteriores) insulae (translated by H.L. Seldon: link)" ${ }^{9}$. A dysfunction of VENs may represent the underlying mechanism of the progressing impairment in episodic migraine ${ }^{50}$; this needs to be specifically investigated with more appropriate techniques at molecular level.

\section{Trajectory of the "pain mode"}

The connectivity between the hypothalamus and the LC was the only significant connection that followed the "pain mode" trajectory. This hypothalamic-pontine connection increased over the pain-free interval and was strongest during the headache phase. Both regions are well-known from the migraine literature on migraine pathophysiology $16,51,52$ and their connectivity was previously found to be altered in migraine ${ }^{53}$.

Hypothalamus. In particular, the hypothalamus has been intensively discussed as the generator of migraine attacks ${ }^{16}$. The clinical features of migraine (e.g. the cyclic behaviour of attacks, prodromal and postdrome symptoms (yawning, fatigue and mood changes), autonomous symptoms (nausea and vomiting), as well as trigger factors, such as certain hormones and psycho-physiological stress point to the hypothalamic contribution of the disease ${ }^{3}$. Imaging studies observed hypothalamic changes during the headache ${ }^{17}$ or prior to the headache (during the prodromal phase; ${ }^{54,55}$. Recently, we have shown that throughout the migraine cycle the hypothalamic connectivity to the limbic system is lowest during the headache attack. We were suggesting a new role of this region for migraine, which is to control limbic circuits. An ultimate breakdown of the potentially inhibiting connectivity could be the trigger for initiating the attack ${ }^{9}$.

Locus coeruleus. Pontine alterations in migraine studies were observed using PET and FMRI 56,57. The LC has been discussed to be involved in arousal and sympathetic and parasympathetic processes 58,59 , the modulation of experimental pain ${ }^{60}$, and the development of chronic pain ${ }^{61}$. A study on rodents found evidence for the involvement of the LC in migraine by selectively destroying noradrenergic projections from this region ${ }^{52}$. Decreased LC noradrenergic signalling inhibited dural-evoked trigeminal activation, which points to a potential antinociceptive function of LC projections on migraine-related pain. Similarly, effective vagus nerve stimulation at $1 \mathrm{~Hz}{ }^{62}$ had a direct effect on the activity of the $L^{2}{ }^{63}$. All of these aspects provide evidence for the fundamental role of the LC during a headache attack.

Connectivity between hypothalamus and locus coeruleus. There is convincing anatomical and functional evidence ${ }^{64}$ for the tight interconnection between the hypothalamus and the LC (for a systematic review see ${ }^{65}$. Several aspects of the function of the thalamic-LC 
connection correspond to autonomic symptoms and point towards an involvement in migraine disease. Particularly through noradrenergic projections, both regions have been suggested to be involved in wakefulness and arousal ${ }^{66}$, stress ${ }^{67,68}$, and appetite ${ }^{69,70}$. However, many of these studies interpret the function of the interconnection between the hypothalamus and the LC either by its anatomical connection and shared neurotransmitter system or by its isolated brain function. Here, we were able to specifically address the relevance of the connectivity in dependence of the phase within the migraine cycle.

Brainstem migraine generator. We also included a pontine brain region that was previously interpreted as the "migraine generator" ${ }^{17,18}$. We included this coordinate into the analysis but did not find any significant connection to any brain area over the migraine cycle for either trajectory. However, our coordinate for the pontine region of interest (LC) is in congruence with the coordinates found by Maniyar and colleagues, particularly for the activity during the headache phase of the migraine attack ${ }^{8}$.

\section{Remarks and Limitations}

The present study has limitations that need to be addressed. Here, we sampled an entire migraine cycle from patients that have natural fluctuations of the cycle length. This inevitably leads to a different number of recordings per participant. In our linear model, this issue can not be solved; the high number of recordings $(n=82)$ would argue against a major impact on the findings.

We were using PCAs to extract the time course of a brain region with the highest explained variance. This approach has been shown to provide reasonable results in previous work ${ }^{71,72}$. However, this approach inevitably neglects further components that may also reflect the cyclic behaviour of cortical connectivity in migraineurs. An alternative approach would require PCAs with concatenated data across all recordings and will result in a much higher number of input processes. In this case, a reasonable cutoff for the number of components for each brain region needs to be determined. However, while the present study allows some individual topographical variability across participants, the topography of PCA components for concatenated data will be identical across sessions.

Furthermore, we investigated predefined and reasonable trajectories across the migraine cycle. These trajectories peak before or during the migraine attack and we assumed linear progress of the trajectories. Although these two trajectories are plausible and supported by previous work, other trajectories might be possible, too. These trajectories could progress non-linearly; cortical processes could be predominantly processed at a different time point within the migraine cycle.

Finally, we consider the recorded headache attack data as invariant throughout all potential follow-up migraine cycles. This particularly applies to the second trajectory, which we labelled "pain mode". However, the extrapolation of the data might not be justified and may explain the limited number of effects in our results. It would have been desirable to have obtained a time series that also includes the follow-up headache attack. However, the existence of a migraine cycle is well-established through neurophysiological and behavioural data and we can assume the same recurring cyclic activity with a universal biological mechanism. In addition, the headache calendar shows that the recorded attacks and the untested follow-up attacks are almost identical in their clinical features. In 
addition, the effects related to diseased and healthy brain states are usually assessed in a single recording of a participant.

\section{Summary and Outlook.}

Most connectivity changes exhibited a linear increase over the pain-free interval with a peak prior to the headache and a "drop" during the headache ("reset mode"). Increasing functional connectivity towards the next attack is suggested to reflect the pathological sensory processing shown in previous behavioural and electrophysiological studies. The collapse of connectivity during the headache can be regarded as a mechanism of normalising cortical processing. Depending on the affected connection, the peak of synchronicity of functional connections during the ictal phase of the migraine cycle may contribute to the variety of symptoms of migraine attacks, such as headache, hypersensitivity to sensory modalities, and autonomous symptoms. These pathological processes are hypothesised to be related to VENs in agranular anterior insular and orbitofrontal brain regions. Future studies using more appropriate molecular techniques are needed to follow up on this hypothesis.

\section{Data availability}

Raw data were generated at the Technische Universität München. The authors confirm that the data supporting the findings of this study are available within the article. Inquiries for additional data are available from the corresponding authors, upon reasonable request.

\section{Funding}

The study has been funded by the Else-Kröner-Fresenius Stiftung (Anne Stankewitz 2014-A85).

\section{Acknowledgements}

We thank Dr Andreas Straube for his comments and suggestions and Dr Stephanie Irving for copy-editing the manuscript.

\section{References}

1. Yeh WZ, Blizzard L, Taylor BV. What is the actual prevalence of migraine? Brain Behav. 2018;8(6):e00950.

2. Blau JN. Migraine: theories of pathogenesis. Lancet. 1992;339(8803):1202-1207.

3. Kelman $L$, Tanis $D$. The relationship between migraine pain and other associated symptoms. Cephalalgia. 2006;26(5):548-553.

4. Judit Á, Sándor PS, Schoenen J. Habituation of Visual and Intensity Dependence of Auditory Evoked Cortical Potentials Tends to Normalize Just Before and During the Migraine Attack. Cephalalgia. 2000;20(8):714-719. doi:10.1111/j.1468-2982.2000.00122.x

5. Kropp P, Gerber WD. Contingent negative variation during migraine attack and interval: evidence for normalization of slow cortical potentials during the attack. Cephalalgia. 1995;15(2):123-128; discussion 78-79. 
6. Ambrosini A, de Noordhout AM, Sándor PS, Schoenen J. Electrophysiological studies in migraine: a comprehensive review of their interest and limitations. Cephalalgia. 2003;23 Suppl 1:13-31.

7. Stankewitz A, Schulz E, May A. Neuronal correlates of impaired habituation in response to repeated trigemino-nociceptive but not to olfactory input in migraineurs: an FMRI study. Cephalalgia. 2013;33(4):256-265.

8. Maniyar FH, Sprenger T, Monteith T, Schankin C, Goadsby PJ. Brain activations in the premonitory phase of nitroglycerin-triggered migraine attacks. Brain. 2014;137(Pt 1):232-241.

9. Stankewitz A, Keidel L, Rehm M, et al. Migraine Attacks as a Result of Hypothalamic Loss of Control. Neurolmage: Clinical. Published online August 16, 2021:102784.

10. Schulte LH, Mehnert J, May A. Longitudinal Neuroimaging over 30 Days: Temporal Characteristics of Migraine. Ann Neurol. 2020;87(4):646-651.

11. Schulte LH, Menz MM, Haaker J, May A. The migraineur's brain networks: Continuous resting state fMRI over 30 days. Cephalalgia. Published online August 23, 2020:333102420951465.

12. Hougaard A, Amin FM, Larsson HBW, Rostrup E, Ashina M. Increased intrinsic brain connectivity between pons and somatosensory cortex during attacks of migraine with aura. Hum Brain Mapp. 2017;38(5):2635-2642.

13. Amin FM, Hougaard $A$, Magon $S$, et al. Altered thalamic connectivity during spontaneous attacks of migraine without aura: A resting-state fMRI study. Cephalalgia. 2018;38(7):1237-1244.

14. Stankewitz A, Schulz E. Intrinsic Network Connectivity Reflects the Cyclic Trajectory of Migraine Attacks. bioRxiv. Published online July 9, 2021:2021.06.30.450593. doi:10.1101/2021.06.30.450593

15. Karsan N, Goadsby PJ. Biological insights from the premonitory symptoms of migraine. Nat Rev Neurol. 2018;14(12):699-710.

16. May A, Burstein R. Hypothalamic regulation of headache and migraine. Cephalalgia. 2019;39(13):1710-1719.

17. Denuelle $M$, Fabre N, Payoux $P$, Chollet $F$, Geraud $G$. Hypothalamic activation in spontaneous migraine attacks. Headache. 2007;47(10):1418-1426.

18. Schulte LH, May A. The migraine generator revisited: continuous scanning of the migraine cycle over 30 days and three spontaneous attacks. Brain. 2016;139(Pt 7):1987-1993.

19. Skorobogatykh $K$, van Hoogstraten WS, Degan $D$, et al. Functional connectivity studies in migraine: what have we learned? J Headache Pain. 2019;20(1):108.

20. Headache Classification Committee of the International Headache Society (IHS). The International Classification of Headache Disorders, 3rd edition. Cephalalgia. 2018;38(1):1-211.

21. Salimi-Khorshidi G, Douaud G, Beckmann CF, Glasser MF, Griffanti L, Smith SM. Automatic denoising of functional MRI data: combining independent component analysis and hierarchical fusion of classifiers. Neuroimage. 2014;90:449-468.

22. Griffanti L, Salimi-Khorshidi G, Beckmann CF, et al. ICA-based artefact removal and accelerated FMRI acquisition for improved resting state network imaging. Neuroimage. 2014;95:232-247.

23. Margulies DS, Böttger J, Watanabe A, Gorgolewski KJ. Visualizing the human connectome. Neuroimage. 2013;80:445-461.

24. Glasser MF, Coalson TS, Robinson EC, et al. A multi-modal parcellation of human cerebral cortex. Nature. 2016;536(7615):171-178.

25. Power JD, Barnes KA, Snyder AZ, Schlaggar BL, Petersen SE. Spurious but systematic correlations in functional connectivity MRI networks arise from subject motion. Neuroimage. 2012;59(3):2142-2154.

26. Stankewitz A, May A. The phenomenon of changes in cortical excitability in migraine is not migraine-specific--a unifying thesis. Pain. 2009;145(1-2):14-17.

27. Schulz E, Stankewitz A, Witkovský V, Winkler AM, Tracey I. Strategy-dependent modulation of cortical pain circuits for the attenuation of pain. Cortex. 2019;113:255-266.

28. Wilkinson GN, Rogers CE. Symbolic Description of Factorial Models for Analysis of Variance. Applied Statistics. 1973;22(3):392. doi:10.2307/2346786

29. Harrison $X A$, Donaldson $L$, Correa-Cano $M E$, et al. A brief introduction to mixed effects modelling and multi-model inference in ecology. PeerJ. 2018;6:e4794.

30. Winkler AM, Ridgway GR, Webster MA, Smith SM, Nichols TE. Permutation inference for the general linear model. Neuroimage. 2014;92:381-397.

31. Winkler AM, Webster MA, Brooks JC, Tracey I, Smith SM, Nichols TE. Non-parametric 
bioRxiv preprint doi: https://doi.org/10.1101/2021.10.18.464798; this version posted October $19,2021$. The copyright holder for this preprint (which was not certified by peer review) is the author/funder, who has granted bioRxiv a license to display the preprint in perpetuity. It is made available under aCC-BY-NC-ND 4.0 International license.

combination and related permutation tests for neuroimaging. Hum Brain Mapp. 2016;37(4):1486-1511.

32. Evrard HC. The Organization of the Primate Insular Cortex. Front Neuroanat. 2019;13:43.

33. Ke J, Yu $Y$, Zhang $X$, et al. Functional Alterations in the Posterior Insula and Cerebellum in Migraine Without Aura: A Resting-State MRI Study. Front Behav Neurosci. 2020;14:567588.

34. Tracey I. Imaging pain. Br J Anaesth. 2008;101(1):32-39.

35. Zhu J, Cheng Q, Chen Y, et al. Transient Delay-Period Activity of Agranular Insular Cortex Controls Working Memory Maintenance in Learning Novel Tasks. Neuron. 2020;105(5):934-946.e5.

36. Smith R, Lane RD, Alkozei $A$, et al. Maintaining the feelings of others in working memory is associated with activation of the left anterior insula and left frontal-parietal control network. Soc Cogn Affect Neurosci. 2017;12(5):848-860.

37. Wager TD, Rilling JK, Smith EE, et al. Placebo-induced changes in FMRI in the anticipation and experience of pain. Science. 2004;303(5661):1162-1167.

38. Fuchs PN, Peng YB, Boyette-Davis JA, Uhelski ML. The anterior cingulate cortex and pain processing. Front Integr Neurosci. 2014;8:35.

39. Tracey I, Mantyh PW. The cerebral signature for pain perception and its modulation. Neuron. 2007;55(3):377-391.

40. Mouraux A, Diukova A, Lee MC, Wise RG, lannetti GD. A multisensory investigation of the functional significance of the "pain matrix." Neuroimage. 2011;54(3):2237-2249.

41. Liang M, Su Q, Mouraux A, lannetti GD. Spatial Patterns of Brain Activity Preferentially Reflecting Transient Pain and Stimulus Intensity. Cereb Cortex. 2019;29(5):2211-2227.

42. Valentini $E$. The role of anterior insula and anterior cingulate in empathy for pain. $J$ Neurophysiol. 2010;104(2):584-586.

43. Coppola G, Pierelli F, Schoenen J. Habituation and migraine. Neurobiol Learn Mem. 2009;92(2):249-259.

44. Tops M, Boksem MAS. A Potential Role of the Inferior Frontal Gyrus and Anterior Insula in Cognitive Control, Brain Rhythms, and Event-Related Potentials. Frontiers in Psychology. 2011;2. doi:10.3389/fpsyg.2011.00330

45. Coppola G, Chen W-T. Neurophysiology of the Migraine Brain. Springer Nature; 2020.

46. Coppola G, Di Lorenzo C, Schoenen J, Pierelli F. Habituation and sensitization in primary headaches. J Headache Pain. 2013;14:65.

47. Ongür D, Ferry AT, Price JL. Architectonic subdivision of the human orbital and medial prefrontal cortex. J Comp Neurol. 2003;460(3):425-449.

48. Allman JM, Tetreault NA, Hakeem AY, et al. The von Economo neurons in the frontoinsular and anterior cingulate cortex. Ann N Y Acad Sci. 2011;1225:59-71.

49. von Economo CF, Koskinas GN. Die Cytoarchitektonik Der Hirnrinde Des Erwachsenen Menschen. J. Springer; 1925.

50. Borsook D, Veggeberg R, Erpelding $N$, et al. The Insula: A "Hub of Activity" in Migraine. Neuroscientist. 2016;22(6):632-652.

51. Holland P, Barloese $M$, Fahrenkrug J. PACAP in hypothalamic regulation of sleep and circadian rhythm: importance for headache. J Headache Pain. 2018;19(1):1-8.

52. Vila-Pueyo M, Strother LC, Kefel M, Goadsby PJ, Holland PR. Divergent influences of the locus coeruleus on migraine pathophysiology. Pain. 2019;160(2):385-394.

53. Moulton EA, Becerra L, Johnson A, Burstein R, Borsook D. Altered hypothalamic functional connectivity with autonomic circuits and the locus coeruleus in migraine. PLoS One. 2014;9(4):e95508.

54. van Oosterhout WPJ, van Opstal AM, Schoonman GG, et al. Hypothalamic functional MRI activity in the initiation phase of spontaneous and glyceryl trinitrate-induced migraine attacks. Eur J Neurosci. 2021;54(3):5189-5202.

55. Karsan N, Bose P, Goadsby PJ. The Migraine Premonitory Phase. Continuum . 2018;24(4, Headache):996-1008.

56. Borsook $\mathrm{D}$, Burstein $\mathrm{R}$. The enigma of the dorsolateral pons as a migraine generator. Cephalalgia. 2012;32(11):803-812.

57. Afridi SK, Giffin NJ, Kaube $\mathrm{H}$, et al. A positron emission tomographic study in spontaneous migraine. Arch Neurol. 2005;62(8):1270-1275.

58. Schwarz LA, Luo L. Organization of the locus coeruleus-norepinephrine system. Curr Biol. 2015;25(21):R1051-R1056. 
59. Liu Y, Rodenkirch C, Moskowitz N, Schriver B, Wang Q. Dynamic Lateralization of Pupil Dilation Evoked by Locus Coeruleus Activation Results from Sympathetic, Not Parasympathetic, Contributions. Cell Rep. 2017;20(13):3099-3112.

60. Hickey L, Li Y, Fyson SJ, et al. Optoactivation of locus ceruleus neurons evokes bidirectional changes in thermal nociception in rats. J Neurosci. 2014;34(12):4148-4160.

61. Taylor BK, Westlund KN. The noradrenergic locus coeruleus as a chronic pain generator. $J$ Neurosci Res. 2017;95(6):1336-1346.

62. Straube A, Ellrich J, Eren O, Blum B, Ruscheweyh R. Treatment of chronic migraine with transcutaneous stimulation of the auricular branch of the vagal nerve (auricular t-VNS): a randomized, monocentric clinical trial. J Headache Pain. 2015;16(1). doi:10.1186/s10194-015-0543-3

63. Zhang Y, Liu J, Li H, et al. Transcutaneous auricular vagus nerve stimulation at $1 \mathrm{~Hz}$ modulates locus coeruleus activity and resting state functional connectivity in patients with migraine: An FMRI study. Neurolmage: Clinical. 2019;24:101971.

64. Samuels ER, Szabadi E. Functional neuroanatomy of the noradrenergic locus coeruleus: its roles in the regulation of arousal and autonomic function part II: physiological and pharmacological manipulations and pathological alterations of locus coeruleus activity in humans. Curr Neuropharmacol. 2008;6(3):254-285.

65. Giorgi FS, Galgani A, Puglisi-Allegra S, Busceti CL, Fornai F. The connections of Locus Coeruleus with hypothalamus: potential involvement in Alzheimer's disease. Journal of Neural Transmission. 2021;128(5):589-613. doi:10.1007/s00702-021-02338-8

66. Berridge CW, Schmeichel BE, España RA. Noradrenergic modulation of wakefulness/arousal. Sleep Med Rev. 2012;16(2):187-197.

67. Ziegler DR, Cass WA, Herman JP. Excitatory influence of the locus coeruleus in hypothalamic-pituitary-adrenocortical axis responses to stress. $J$ Neuroendocrinol. 1999;11(5):361-369.

68. Hernández-Pérez OR, Hernández VS, Nava-Kopp AT, et at. A Synaptically Connected Hypothalamic Magnocellular Vasopressin-Locus Coeruleus Neuronal Circuit and Its Plasticity in Response to Emotional and Physiological Stress. Front Neurosci. 2019;13:196.

69. Sciolino NR, Mazzone CM, Plummer NW, Evsyukova I. A role for the locus coeruleus in the modulation of feeding. BioRxiv. Published online 2019. https://www.biorxiv.org/content/10.1101/2019.12.18.881599v1.abstract

70. Ammar $A A$, Södersten $P$, Johnson $A E$. Locus coeruleus noradrenergic lesions attenuate intraoral intake. Neuroreport. 2001;12(14):3095-3099.

71. Schulz E, Stankewitz A, Winkler AM, Irving S, Witkovský V, Tracey I. Ultra-high-field imaging reveals increased whole brain connectivity underpins cognitive strategies that attenuate pain. Elife. 2020;9. doi:10.7554/eLife.55028

72. Mayr A, Jahn P, Deak B, Stankewitz A, Devulapally V. Individually Unique Dynamics of Cortical Connectivity Reflect the Ongoing Intensity of Chronic Pain. bioRxiv. Published online 2021. https://www.biorxiv.org/content/10.1101/2021.06.30.450553.abstract

\section{Figure legends}

Fig. 1. I Time course of individual recordings between two migraine attacks. Solid vertical lines indicate recording days. The dashed line at the end of the cycle represents the day of the subsequent migraine attack (not recorded) at which the recordings for the subjects were discontinued. The red lines indicate the attack days. The vertical black lines in between the attacks represent the recordings in the inter-ictal period. We recorded only one cycle per subject. The number of days between the first recorded migraine attack and the subsequent migraine attack is shown on the right and indicates the different lengths of each patient's migraine cycle.

Fig. 2 | Time series of migraine-related brain processes. Two hypothetical time series (trajectories) of migraine-related brain processes were modelled in the statistical analysis. In the first time series (upper part) the brain processes drop during the headache attacks; 
the brain processes would be "reset" during attacks, then would resemble the processes on the day after the attacks. In the second time series (lower part), the brain processes would reach their minimum/maximum during the attacks and are similar to the days before attacks. These processes could be used as a biomarker for an impending migraine attack. The figure is intended to illustrate the cyclic nature of migraine attacks and the time-varying magnitude of two potential brain processes; we recorded only one migraine cycle (dark grey area).

Figure 3a | confusion matrix on migraine cycle-related connectivity changes across all 425 brain regions. The left matrix (A) shows the data for trajectory 1 (reset mode) that has its peak at a pain-free time point just before the migraine attack. All of these trajectories show an increase of connectivity over time with a drop during the headache attack. There was no decreasing connectivity towards a minimum at the time point just before the attack. The right matrix (B) has only a single significant pixel that represents the highest connectivity between the hypothalamus and the LC during a migraine attack (pain mode).

Figure 3b | Cycle-related connectivity changes following the "reset mode" trajectory. The upper part shows a circular plot (link) representing all significant connectivities.

Figure 3c | Cycle-related connectivity changes following the "reset mode" trajectory. The figure shows the connected brain regions. The colour coding in the upper part indicates the number of connections with the highest number for the area $47 \mathrm{~m}$ (12) and the aAIC (10). 


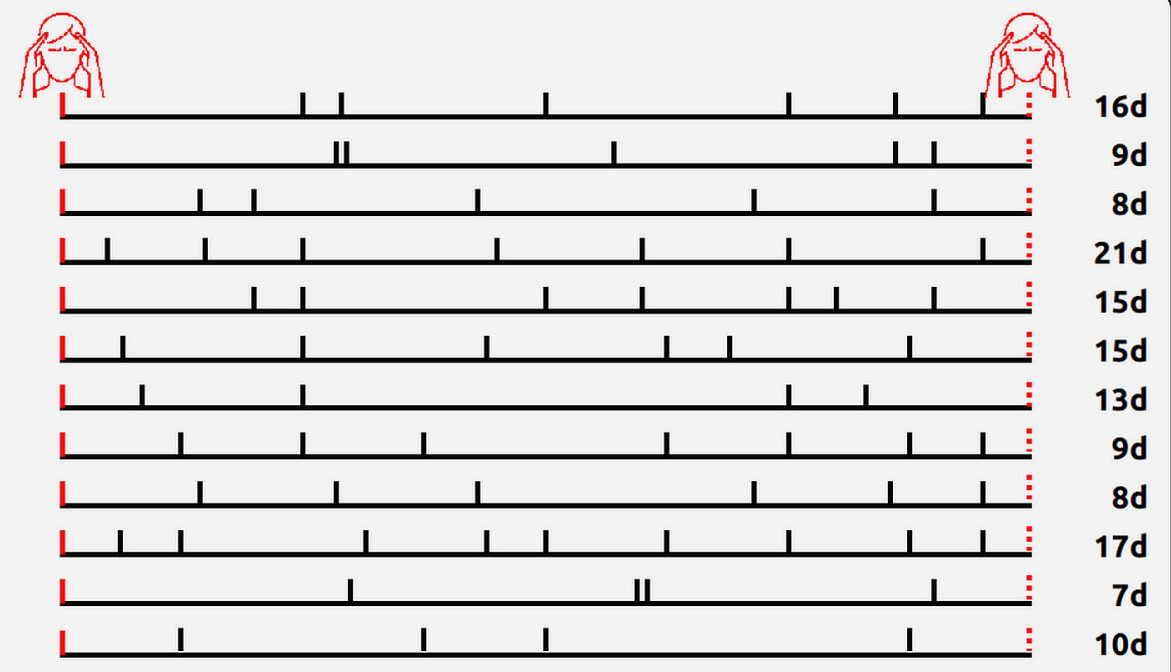




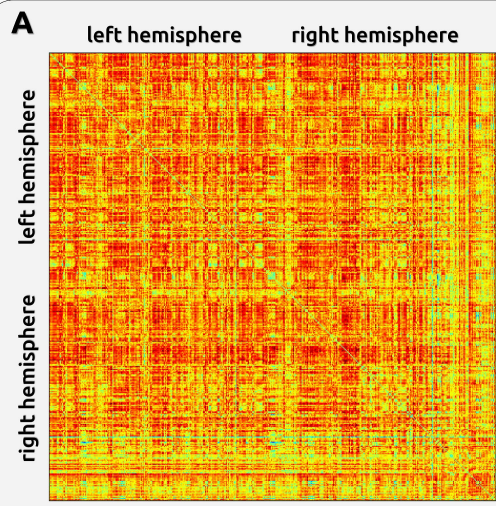

B

reset mode

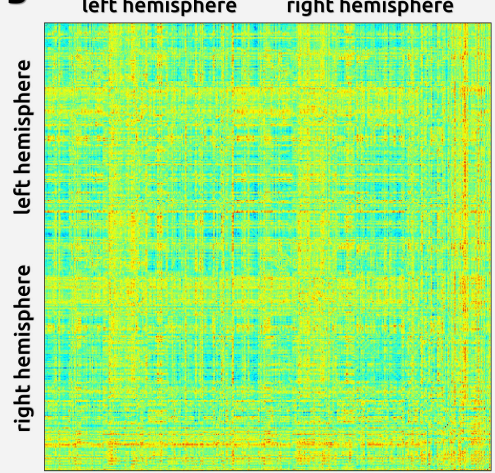




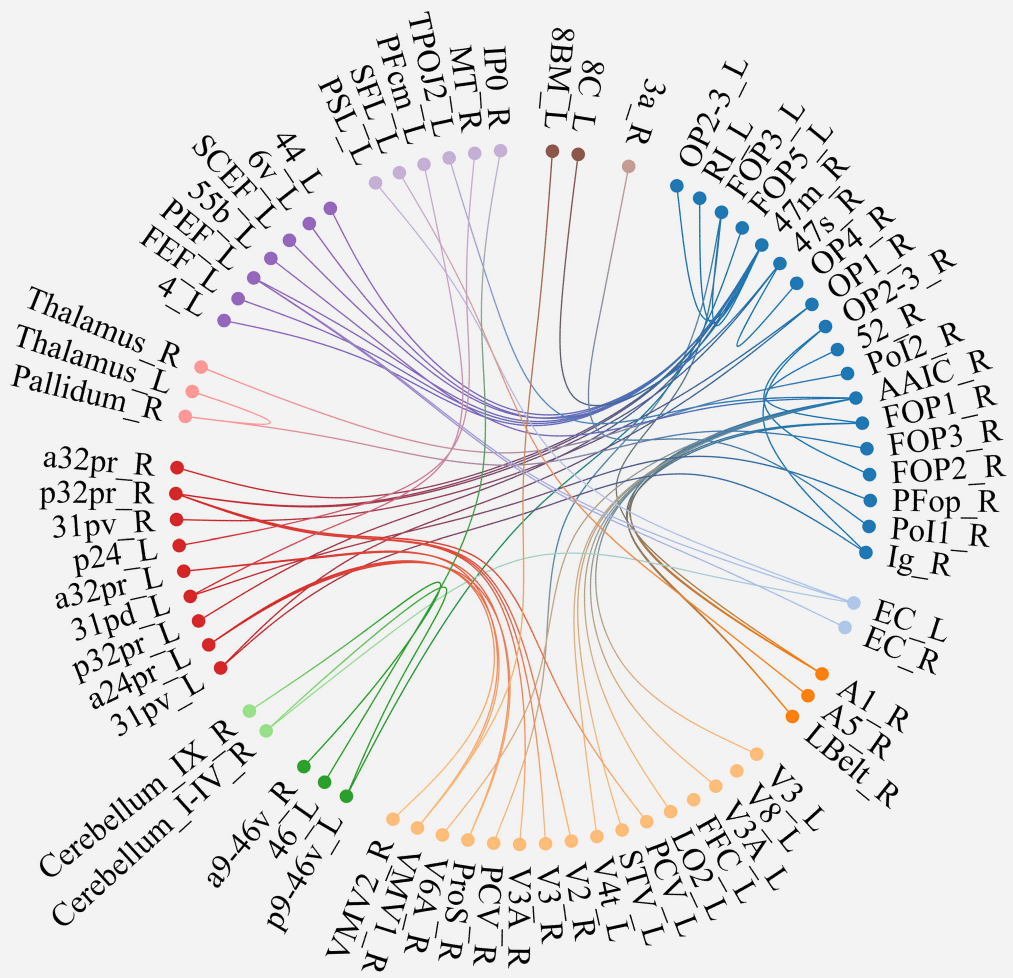

insular entorhinal auditory visual

\section{prefrontal} cerebellar cingulate thalamus, basal motor temporo-parietal 

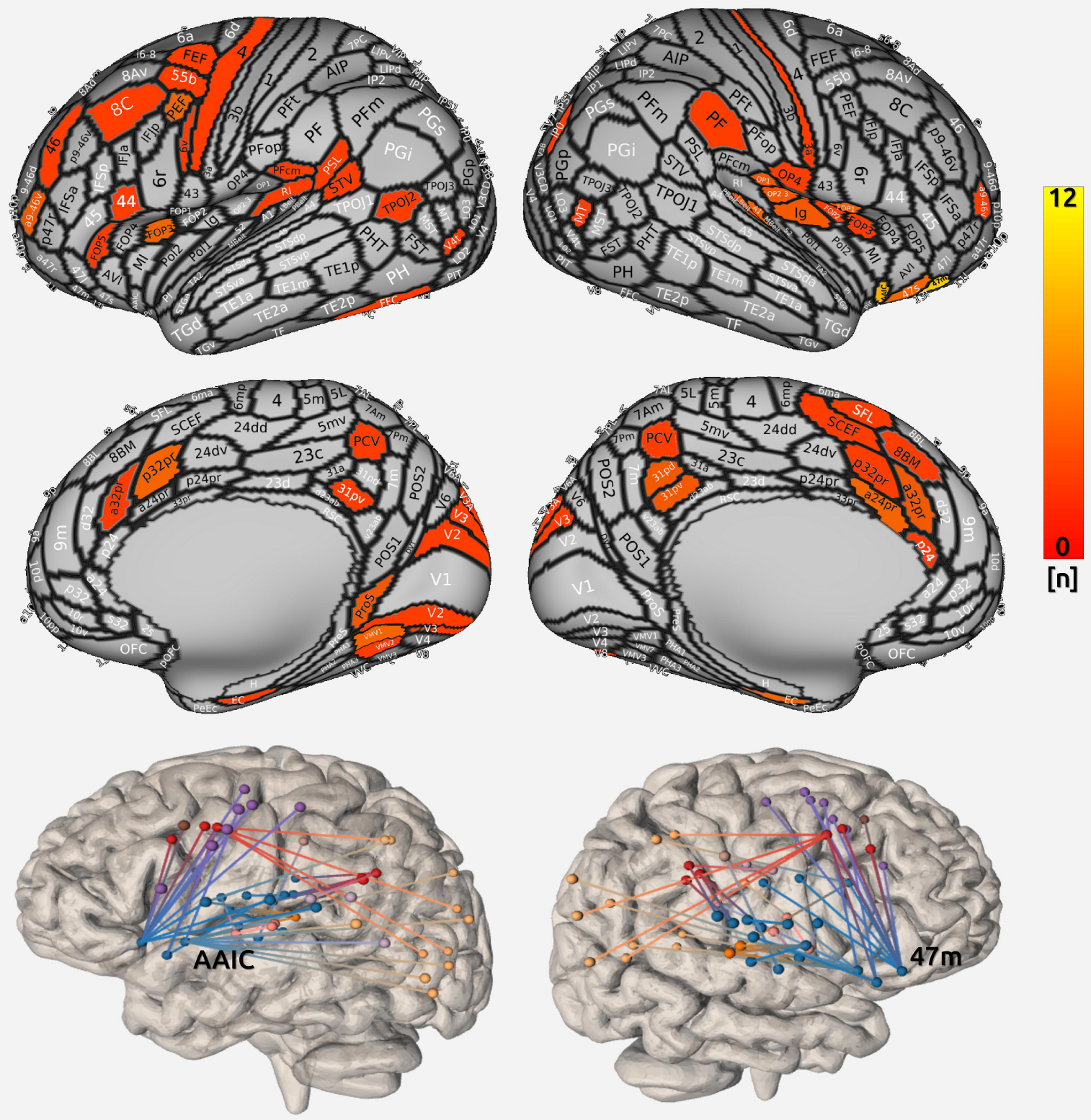

$\left.\begin{array}{l}\text { insular } \\ \text { entorhinal }\end{array} \begin{array}{l}\text { auditory } \square \begin{array}{l}\text { prefrontal } \\ \text { visual }\end{array} \\ \text { cerebellar }\end{array} \square \begin{array}{l}\text { cingulate } \\ \text { thalamus, basal }\end{array}\right\} \begin{aligned} & \text { motor } \\ & \text { temporo-parietal }\end{aligned}$ 